\title{
Strates
}

STRATES Matériaux pour la recherche en sciences sociales

$15 \mid 2008$

Union européenne - Voisinages. La quête d'une intégration régionale

\section{Politique de cohésion et coopération territoriale avec le voisinage. À la recherche d'une plus grande coordination?}

Nicolas Gaubert et Yann Richard

\section{CpenEdition}

Journals

Édition électronique

URL : http://journals.openedition.org/strates/6528

DOI : $10.4000 /$ strates.6528

ISSN : 1777-5442

Éditeur

Laboratoire Ladyss

Édition imprimée

Date de publication : 1 janvier 2008

Pagination : 37-58

ISSN : 0768-8067

Référence électronique

Nicolas Gaubert et Yann Richard, «Politique de cohésion et coopération territoriale avec le voisinage

À la recherche d'une plus grande coordination? », Strates [En ligne], 15 | 2008, mis en ligne le 25

janvier 2013, consulté le 08 septembre 2020. URL : http://journals.openedition.org/strates/6528 ;

DOI : https://doi.org/10.4000/strates.6528

Ce document a été généré automatiquement le 8 septembre 2020.

Tous droits réservés 


\title{
Politique de cohésion et coopération territoriale avec le voisinage. À la recherche d'une plus grande coordination?
}

\author{
Nicolas Gaubert et Yann Richard
}

1 L'économie de l'Union européenne est ouverte sur l'espace mondial. Le territoire de l'Union n'est pas indifférent aux grandes évolutions qui interviennent dans le monde. Par exemple, le processus de mondialisation profite essentiellement aux grandes régions métropolitaines. Ces régions s'enrichissent plus vite que la moyenne communautaire, alors que les régions les plus pauvres, généralement éloignées des principales métropoles, s'enrichissent sensiblement moins vite. À plus grande échelle, il existe des relations fortes entre l'Union européenne (l'UE) et son environnement régional, dans les deux sens. Les régions des États membres de l'UE sont en interaction avec celles des pays voisins, principalement les régions périphériques situées le long des frontières externes de l'UE (Didelon et Grasland, 2006, Beckouche et al., 2008). À titre d'exemple, on peut citer les régions frontalières de l'Union qui vivent en partie de leurs échanges avec les pays tiers voisins (commerce et flux migratoires transfrontaliers entre la Pologne orientale et l'Ukraine ou entre la Roumanie orientale et la Moldavie). Ces interactions ne peuvent que se renforcer à moyen terme, sous l'effet du processus de régionalisation des échanges dans le monde (Beckouche, Richard, 2005, Gaulier et al., 1998, Gemdev, 1994, Siroën, 1995, 2000).

Il semble que l'UE n'ait pas encore pris la mesure de cette évolution. Avec l'affirmation de la politique européenne de cohésion, à la fin des années 1980, se sont amorcées les premières réflexions sur l'aménagement $d u$ territoire dans une perspective européenne. Les grands documents produits dans le cadre de cette réflexion montrent un espace communautaire fermé sur lui-même, comme si les régions de l'UE étaient indifférentes à ce qui se passe juste de l'autre côté de la frontière. Malgré les efforts consentis pour améliorer la coopération transfrontalière avec les régions de pays tiers, 
la politique de cohésion est conçue exclusivement dans un cadre européen fermé. L'hypothèse qui préside à cet article est qu'il existe, comme l'ont montré les travaux classiques de sociologie des organisations et de science politique (Simon, 1957; Allison et al., 1999), une pluralité d'acteurs institutionnels avec des critères et une rationalité propres. Les organisations au sein de la Commission européenne obéissent à leurs propres logiques (Joanna et Smith, 2002) : dès lors, des représentations territoriales et des pratiques de développement sont susceptibles d'être appliquées de façon différenciée. En mettant l'accent sur l'autonomisation et la différenciation des acteurs communautaires - la direction générale de la politique régionale (DG Regio) et la direction générale des relations extérieures (DG Relex) - l'enjeu est de saisir la lecture par la Commission de certains déséquilibres régionaux au sein de l'espace communautaire. Et par là d'étudier la façon dont cela se traduit dans la mise en œuvre d'instruments pour réduire de tels déséquilibres.

3 La recherche d'une meilleure cohérence entre politique régionale et Politique de voisinage s'est traduite par la mise en œuvres récente de nouveaux dispositifs réglementaires. Les nouveaux règlements des fonds structurels et des instruments de la coopération extérieure (Instrument d'aide de pré-adhésion et instrument européen de voisinage et de partenariat) apportent de réelles améliorations aux outils existants (Interreg III A et III B, Tacis et Phare CTF). Ils confirment la tendance engagée en 2003 et 2004 pour relever la qualité des relations avec les pays voisins, en particulier pour la coopération à l'échelle locale. Toutefois, malgré l'ampleur du travail réalisé par la Commission européenne et les États membres, ces outils présentent des insuffisances qui causeront certainement de nouveaux blocages à court et moyen termes. Quels sont les principaux apports de nouveaux règlements publiés à la fin de l'année 2006 pour la réforme de la politique de cohésion et de la Politique de voisinage? Quels blocages risquent d'émerger rapidement?

\section{Une représentation fermée du territoire de l'UE}

4 La politique de cohésion de l'UE vise à réduire les disparités de niveau de développement entre les régions des États membres. Mais tous les grands documents stratégiques qui président à l'élaboration de cette politique et à sa mise en œuvre reposent sur une vision fermée de l'espace communautaire, comme si, pour les responsables de la politique régionale, il n'existait pas de relations entre le territoire des États membres et celui des voisins au sens large. Or il existe non seulement des relations entre l'UE et son environnement régional, ainsi qu'avec le reste du monde, mais aussi des interactions fortes. En d'autres termes, ce qui se passe à l'extérieur a nécessairement des répercussions sur les dynamiques régionales internes de l'Union elle-même. Le calibrage inadéquat de la politique régionale, qui est l'objet de cette première partie, nuit sans doute à son efficacité. Cette première partie s'appuie sur l'analyse des grands textes fondamentaux de la politique européenne de cohésion. Ces textes sont généralement plus incitatifs voire indicatifs qu'impératifs. Les États membres ne sont pas juridiquement liés par le Schéma de développement de l'Espace Communautaire ou par les Rapports sur la cohésion. Néanmoins, ces documents ne sont pas sans valeur. Leur statut dépasse le simple affichage de slogans formels. Ils sont le fruit d'un travail de réflexion commun et traduisent l'émergence progressive d'une culture et de pratiques communes en matière d'aménagement du territoire et de 
développement régional (Albrechts et al., 2003). En retour, ils dessinent des axes stratégiques qui pèsent nécessairement sur les choix faits par les pays membres en matière d'aménagement. La politique européenne de cohésion est le deuxième poste budgétaire de l'UE (plus de 40 milliards d'euros par an). Ce poids financier important donne un pouvoir certain aux services de la Commission pour promouvoir le contenu des documents étudiés ci-dessous. Les territoires des pays membres ont d'autant plus de chance d'obtenir des financements sur les fonds structurels que leurs projets s'inscrivent dans les priorités formulées par les documents tels le Sdec et l'Agenda territorial. Enfin et surtout, ces documents communautaires présentent un contenu qui est en cohérence avec les règlements des fonds structurels. Or ces derniers ont un caractère impératif.

5 C'est au début des années 1990 qu'une planification spatiale stratégique s'est développée, notamment à l'échelon européen (Healey, 2004). La nécessité d'une meilleure coordination des politiques publiques, la recherche de renforcement de la " compétitivité territoriale » (Camagni, 2006), l'objectif de développement durable ou encore la prise en compte d'une meilleure accessibilité, apparaissent au rang des priorités déployées à plusieurs échelles.

6 Au niveau communautaire, le discours sur l'espace s'est très largement développé sous les auspices de la Direction générale pour le Développement régional (DG Regio). La nature de l'intervention de la Commission européenne s'est transformée : les objectifs ont été étendus afin de prendre en compte une dimension plus stratégique de l'aménagement et du développement des territoires (Richarson et Olsen, 2004). Cette dimension "stratégique » du développement des territoires renvoie ici à un processus social dont la vocation est de développer et d'entretenir des relations territoriales insufflées par un savoir partagé et cimenté par des cadres de référence communs (Healey et al., 1997).

\section{Une prise en compte de l'extérieur très relative par la Commission européenne}

7 Le développement des instruments de politique de cohésion a conduit de façon progressive la Commission à intervenir dans le champ de l'aménagement du territoire. Comme les institutions européennes ne possédaient pas de compétences juridiques, il n'y a jamais eu d'intervention explicite à l'échelon communautaire mais la pratique a causé l'émergence d'une forme de planification spatiale. Ainsi, l'article 10 du règlement du Fonds européen de Développement régional (Feder), adopté en 1988 et repris sans modification en 1993, permet à la Commission de rassembler les éléments nécessaires à l'établissement d'un schéma prospectif d'utilisation de l'espace communautaire et de financer les projets pilotes qui constituent des incitations à la réalisation d'infrastructures ainsi que d'autres mesures spécifiques ayant un intérêt communautaire marqué. C'est dans ce cadre qu'ont été lancés, à l'initiative de la Commission européenne, les rapports "Europe 2000 » (1991) et "Europe $2000+$ » (1994). Ces deux rapports mettent en relief la nécessité de mettre en œuvre un développement équilibré du «territoire communautaire ». Ils préfigurent, sur le plan intergouvernemental, la mise en place du schéma de développement de l'espace communautaire $(\mathrm{SDEC})$ avec une prise en compte très réduite de la dimension extérieure. 
Concomitamment à ces initiatives expérimentales dans le domaine de l'aménagement du territoire, la Commission européenne a identifié les évolutions et les priorités en termes de politiques structurelles au travers des rapports sur la cohésion. Véritables états des lieux des grandes tendances territoriales de l'UE, les Rapports sur la cohésion, produits par la Commission européenne (à un rythme triennal) concentrent de la même façon l'attention sur l'espace communautaire uniquement. Ainsi, le premier rapport sur la cohésion (Commission européenne, 1996), aborde essentiellement la question du transfrontalier à l'échelle de l'ensemble des États membres :

Il n'y a guère de priorité plus importante dans le processus d'intégration européenne que l'élimination des barrières traditionnelles entre États membres qui introduisent des distorsions dans les structures économiques et réduisent les possibilités ouvertes, notamment les communautés frontalières. ${ }^{1}$

9 Les perspectives de l'élargissement à dix nouveaux États membres mettent cependant en lumière l'importance d'appréhender le développement territorial au-delà des frontières de l'UE. Le deuxième rapport sur la cohésion (2001) met en relief cet aspect :

L'élargissement rendra nécessaire de nouvelles actions transfrontalières pour encourager la coopération entre les anciens et les nouveaux États membres de l'Union et aider les régions des États qui, à l'est et au sud, partagent des frontières avec des pays tiers, y compris sur le pourtour méditerranéen. ${ }^{2}$

La cohésion est présentée uniquement sous l'angle de la politique régionale européenne. En concevant une politique de cohésion régionale qui prime sur l'objectif de convergence macroéconomique, le deuxième rapport témoigne d'une conception très large de la politique régionale européenne, indépendamment de l'objectif de réduction des inégalités interrégionales de développement au sein des États membres (Davezies, 2002). Cette approche fait ainsi l'impasse sur la mise en place d'instruments destinés à être mis en œuvre de façon différenciée entre les régions, notamment pour les espaces transfrontaliers (UE - pays voisins). Elle occulte par ailleurs l'importance d'une coordination des politiques sectorielles ayant une finalité territoriale pourtant revendiquée (Commission européenne, 2001).

11 Les frontières extérieures de l'UE sont abordées dans le troisième rapport sur la cohésion (Commission européenne, 2004) au prisme de l'initiative Intereg. Ce programme d'initiative communautaire est présenté comme un moyen de développer les échanges transfrontaliers à l'intérieur de l'UE et avec les pays voisins :

Une partie de la valeur ajoutée des programmes Interreg IIA tient à leur contribution à l'instauration et au renforcement d'une culture transfrontalière à la fois à l'intérieur et entre l'Union et les pays voisins. ${ }^{3}$

12 Ce programme a permis de générer une nouvelle forme d'espace de gouvernance transnational avec une démarche "par le bas" (bottom-up) associant les décideurs locaux (Christiansen et Jorgensen, 2000). Mais le développement des espaces transfrontaliers au-delà des frontières de l'UE est appréhendé de façon restrictive à la fois en termes d'ambition - la perspective extérieure reste peu évoquée - et de moyens. Dans le 4- rapport sur la cohésion, la coopération transfrontalière à l'intérieur de l'UE et dans les régions situées le long des frontières extérieures de l'UE est abordée sans davantage de développements (Commission européenne, 2007). En outre, le rapport évoque cette question sur le mode d'une simple déclaration de principe car les effets territoriaux de la coopération aux frontières extérieurs sont encore peu tangibles :

De nombreuses années de programmes transfrontaliers ont amélioré la coopération entre régions frontalières à l'intérieur de l'UE-15 [...]. Mais ces nouvelles frontières 
intérieures ne sont pas encore perméables et les flux transfrontaliers sont nettement moindres.

Renforcer la perméabilité tant physique qu'administrative de ces frontières facilitera le flux de personnes et de biens entre ces régions et rapprochera le niveau des échanges économiques du potentiel économique de ces régions. Ce type d'activité de coopération est même plus important pour les régions situées aux frontières extérieures de l'UE. ${ }^{4}$

13 Ainsi, si les discours communautaires reconnaissent l'importance de la prise en compte des frontières extérieures, les modalités précises de la coopération entre l'UE et les pays voisins en matière de planification restent à développer. Ces difficultés peuvent renvoyer à des conceptions de la coopération transfrontalière dont le caractère resterait essentiellement interne à l'UE et demeurerait ainsi un instrument de renforcement des échanges intracommunautaires. La coopération transfrontalière à l'intérieur de l'espace européen suppose un processus d'homogénéisation des systèmes juridiques, administratifs et politiques des États membres (O'Dowd, 2002). La création de ces nouveaux espaces de coopération (Perkmann, 1999), dépassant le lien entre frontières et identités nationales (Paasi, 1998), est de plus encore récente. L'affirmation de cette coopération met surtout en relief la dimension interne de ce processus, évolution qui se retrouve dans le cadre des réflexions sur l'aménagement du territoire dans une démarche intergouvernementale.

\section{Une approche très limitée dans le cadre intergouvernemental}

14 Si l'UE n'a pas de compétence formelle sur le plan de l'aménagement du territoire, les États membres en coopération avec la Commission européenne ont progressivement soulevé la nécessité de coordonner les politiques communautaires en vue d'un développement territorial à l'échelle communautaire.

Résultant d'un processus engagé sous la présidence française de l'UE en 1989, le Schéma de développement de l'espace communautaire (SDEC) répond à l'objectif de mettre en place une réflexion stratégique en vue de permettre le développement équilibré au sein de l'espace européen. Les principes directeurs du document étaient de trois ordres : le développement spatial et polycentrique et les nouvelles relations ville-campagne; l'accès équivalent aux infrastructures et au savoir ; la gestion prudente du patrimoine naturel et culturel. Ce document - qui n'a aucun aspect contraignant - a été adopté en mai 1999. Dans un contexte où les perspectives d'élargissement de l'UE sont au cœur de l'agenda politique, le Sdec aborde notamment la question des frontières extérieures dans le cadre des axes structurants :

Le renforcement des villes et des régions aux frontières extérieures de l'UE: des politiques pour le développement des "villes portes", de l'infrastructure multimodale pour les corridors européens, d'un accès équivalent aux services de télécommunications et d'une bonne accessibilité intercontinentale sont susceptibles de renforcer le rôle des régions et des villes situées le long des frontières extérieures. Ceci est valable autant pour le processus d'élargissement que pour l'approfondissement des relations avec des pays tiers, que ce soit vers le sud ou vers d'autres régions économiques d'importance mondiale. ${ }^{5}$

16 Il reste que, dans les faits, une procédure de définition des priorités européennes à l'échelle des grands ensembles régionaux dans le domaine des réseaux transeuropéens a rencontré une concrétisation difficile (Rees \& Deblanc, 2004). De plus, les 30 projets prioritaires arrêtés en avril 2004 se concentraient essentiellement sur l'UE comprenant 
les dix nouveaux États membres. Le rapport ${ }^{6} \mathrm{du}$ groupe de haut niveau présidé par Mme Loyola de Palacio (novembre 2005) prône une amélioration des liaisons de transports avec les pays voisins de l'UE. Ce rapport a très largement nourri la communication de la Commission relative à l'extension des axes transeuropéens de transports aux pays voisins (Commission européenne, 2007).

\section{Planification territoriale et voisinage : l'évolution pour la période 2007-2013}

17 La politique de cohésion pour la période 2007-2013 comporte désormais un objectif de coopération territoriale. La nécessité d'une cohérence accrue en matière d'actions transfrontalières est évoquée dans les orientations stratégiques communautaires. En fixant les grands objectifs, ces orientations stratégiques s'insèrent dans la nouvelle architecture de la politique de cohésion, qui se décline également au niveau national par l'intermédiaire de stratégies nationales de développement, négociées avec la Commission européenne.

Les frontières extérieures sont mentionnées de la façon suivante :

Il faut accorder une attention particulière aux défis et opportunités qui résultent de la modification des frontières extérieures de l'Union à la suite de l'élargissement. À cet égard, il est nécessaire de favoriser des actions transfrontalières cohérentes qui encouragent l'activité économique de part et d'autre des frontières et éliminent les obstacles au développement. À cette fin, la politique de cohésion et le nouvel instrument européen de voisinage et de partenariat ainsi que, le cas échéant, le nouvel instrument de pré-adhésion, doivent créer un cadre cohérent pour de telles actions. $^{7}$

Sur le plan intergouvernemental, les ministres responsables pour l'aménagement du territoire ont décidé d'introduire la dimension territoriale dans le processus de Lisbonne lors de la réunion ministérielle informelle sur la cohésion territoriale qui s'est tenue à Rotterdam en novembre 2004 (Faludi et Waterhout, 2005). Dans le cadre du processus de l'« Agenda territorial » dont les objectifs sont de fixer les grands enjeux en matière de cohésion territoriale, adoptée le 25 mai 2007, la mention explicite aux pays voisins est absente. Le document de référence, qui appuie les réflexions dans le domaine du développement territorial, «L'état et les perspectives du territoire de l'UE », évoque également le voisinage de façon lapidaire :

Le succès de la stratégie de Lisbonne dépendra non seulement de l'intégration régionale interne de l'Europe mais également de l'intégration de l'Europe dans son voisinage. $^{8}$

Là encore l'insistance sur les potentialités des seuls États membres révèle une représentation fermée du territoire européen. La prise en compte des interactions qui existent entre celui-ci et le territoire des pays proches est ignoré. Des perspectives s'ouvrent cependant avec le souhait exprimé par les ministres responsables de l'aménagement du territoire de prendre en compte les aspects relatifs à la politique européenne de voisinage dans la mise en œuvre de l'Agenda territorial, considérée comme un des « dossiers clés d'un point de vue territorial »". 


\section{Les nouveaux fonds structurels et la nouvelle politique européenne de voisinage apporteront-ils des améliorations ?}

Depuis le début des années 2000, l'UE tente d'améliorer les outils institutionnels dont elle dispose pour encadrer et développer les relations avec les pays voisins. Elle a été proposée en 2002 au conseil européen de Copenhague ; ses objectifs ont été énoncés par Romano Prodi en 2003, dans une communication de la Commission, et en 2004 dans un document de stratégie ; elle a été mise en œuvre en 2004. Depuis le $1^{\mathrm{er}}$ janvier 2007, ce sont des versions rénovées de la Politique de voisinage et de la politique régionale qui sont en vigueur. De réelles améliorations ont été apportées dans les nouveaux textes réglementaires. Elles sont une première réponse à des difficultés anciennes de tous ordres relevées par la Commission elle-même et par d'autres institutions européenne ${ }^{10}$. Pour autant, on peut se demander si elles sont le signe d'une plus grande prise en compte des interactions avec le voisinage et de leur impact éventuel sur le territoire de l'UE. Trois aspects importants des nouveaux règlements seront présentés dans cette partie.

Les anciens programmes d'aide de l'UE pour les pays proches (jusqu'en 2006).

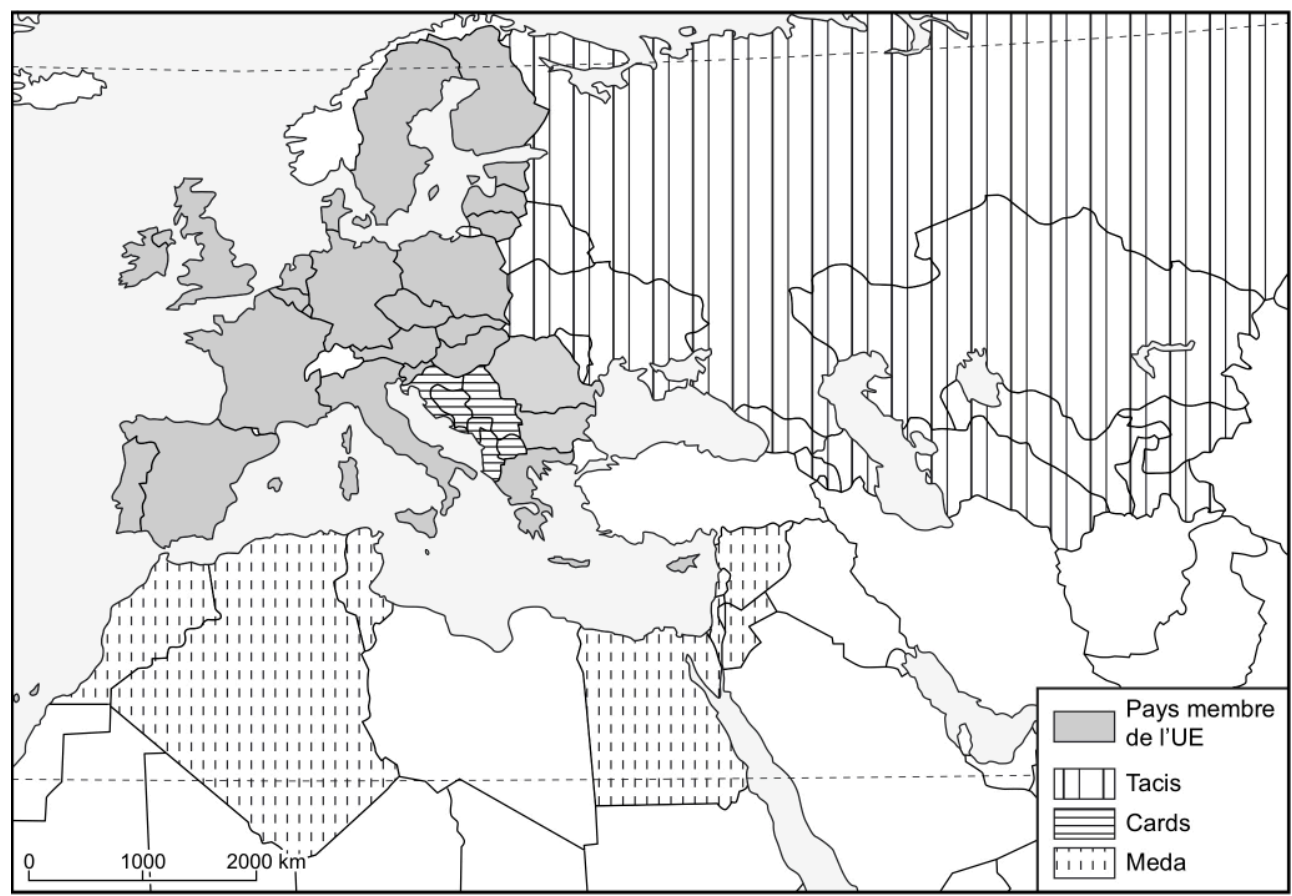




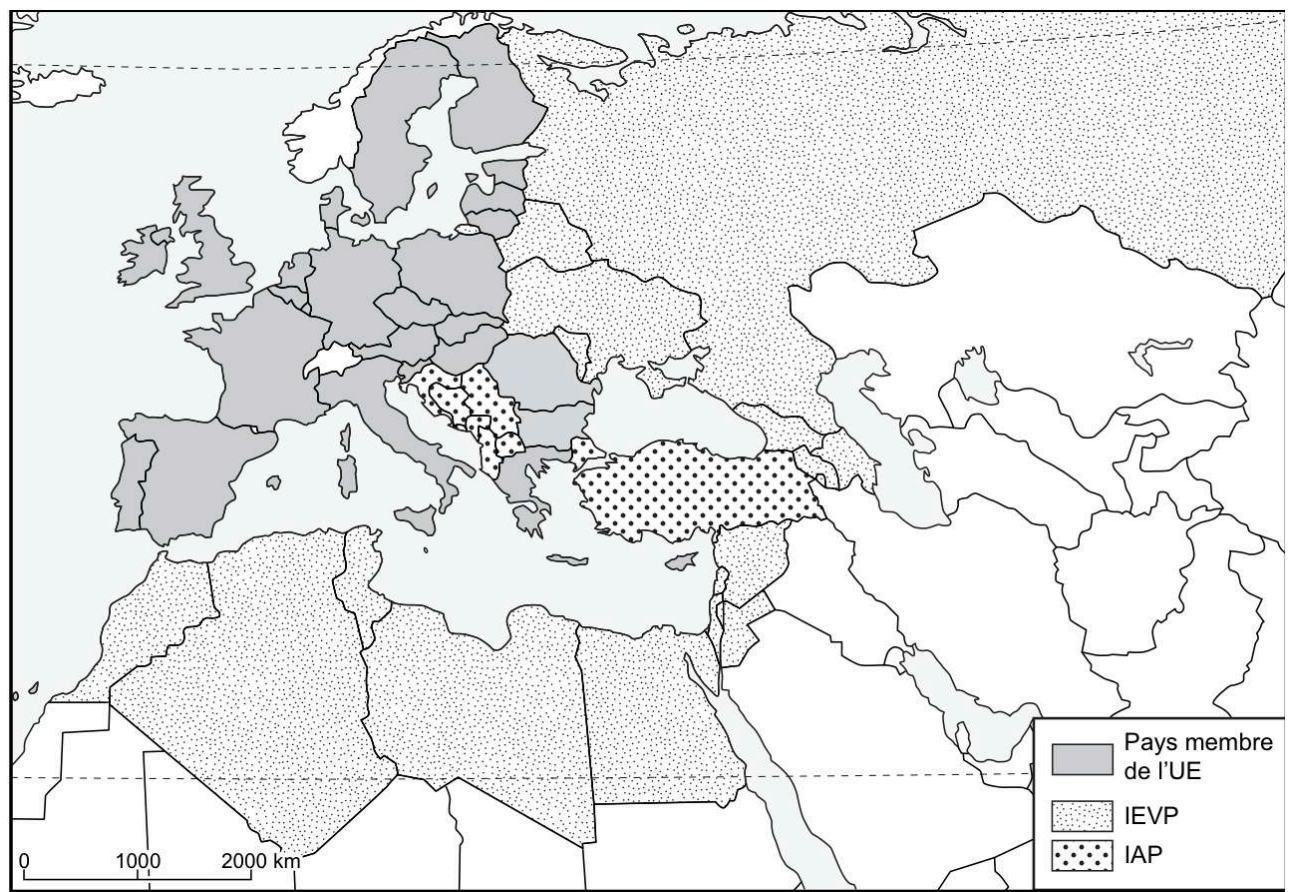

\section{Une plus grande souplesse géographique pour l'utilisation des fonds structurels}

Dès les années 1990, l'efficacité des outils de la coopération transfrontalière le long des frontières externes de l'UE avait été amoindrie par l'inadéquation entre les divers instruments financiers mis en œuvre. Les procédures administratives et financières propres à chacun de ces instruments étaient difficiles à articuler. Cet état de fait a été relevé, entre autres, par la Commission et par la Cour des Comptes européenne dans diverses communications :

En ce qui concerne les frontières extérieures et la coopération avec les pays tiers, des difficultés supplémentaires sont apparues en particulier en raison de structures administratives lacunaires et du manque de ressources dans ces pays. En outre, on a constaté des problèmes de coordination entre les programmes Interreg et ceux des instruments communautaires de politique extérieure tels que Phare, Tacis et MEDA, à cause de discordances dans les règles opérationnelles. ${ }^{11}$

En réponse à certaines de ces difficultés, la Commission a publié un "Manuel pour la coordination du financement Tacis et Interreg» en 2001, pour faciliter le financement de projets de coopération entre pays membres de l'UE et pays de la CEI. Ce manuel est signé par les deux commissaires en charge de la Politique régionale et des Relations extérieures. Cette double signature atteste d'une prise de conscience : pour renforcer réciproquement leur efficacité, certains outils de la politique régionale et des relations extérieures doivent être mieux articulés. Pour autant, rien ne dit dans ce manuel que les deux administrations avaient pris conscience de la nécessité de travailler en plus grande coordination à différentes échelles. Il propose des améliorations significatives seulement à l'échelle locale et régionale (coopération transfrontalière et transnationale). ${ }^{12}$ 
Parallèlement, la Commission a mis en œuvre les programmes de voisinage en 2004, dont l'idée avait été lancée dans divers documents préalables. ${ }^{13}$ Ce nouvel instrument pose les conditions d'une réelle coopération de tous les pays impliqués dans la coopération transfrontalière et transnationale (pays membres de l'UE et pays tiers voisins) le long des frontières externes de la Communauté. Cela signifie que tous les projets de coopération doivent être élaborés en commun par des acteurs situés des deux côtés de la frontière, et que des structures communes de gestion, de suivi et de conseil sont mises en œuvre. C'est une structure commune (le Joint Selection Committee) qui choisit les projets financés. Chacun des organes communs ainsi créés (Joint Technical Secretariat, Joint Selection Committee et Joint Monitoring Committee) doit être composé par des personnes venues de l'ensemble des pays impliqués. Selon les membres du secrétariat technique conjoint de Riga, interviewés en 2005 et 2006, les programmes de voisinage ont apporté une réelle amélioration. Ils ont obligé les acteurs de la coopération à une plus grande collaboration de l'amont à l'aval, depuis la conception des projets jusqu'à leur mise en œuvre. Les problèmes d'articulation des instruments financiers et des procédures réglementaires n'ont pas été totalement éliminés, mais la procédure conjointe oblige les acteurs à proposer des projets qui auront un impact des deux côtés de la frontière externe de l'Union.

\section{L'apport des nouveaux textes réglementaires dans les nouvelles réglementations : l'évolution du Feder et de la Politique de voisinage}

Les nouveaux règlements des instruments de la politique de cohésion et de la politique des relations extérieures ont été conçus pour poursuivre les efforts engagés au début des années 2000. Ils confirment en particulier l'effort déjà fait pour leur donner une plus grande souplesse d'utilisation. Il est désormais plus facile d'utiliser les crédits du Fonds européen de Développement régional sur le territoire des pays tiers, à condition que le projet ainsi financé ait un impact positif sur le territoire communautaire. Jusqu'en 2006, l'utilisation de crédits Feder à l'extérieur de l'UE n'était pas interdite mais elle était très strictement limitée à des dépenses non opérationnelles (frais de déplacement, voyages, etc.). ${ }^{14}$

Parallèlement, la définition des zones éligibles à l'utilisation des crédits Feder est plus large qu'auparavant, afin d'augmenter l'impact des projets de développement dans les zones directement concernées.

Dans le cadre de la coopération transfrontalière, le Feder peut financer, [...] jusqu'à concurrence de $20 \%$ du montant de sa contribution au programme opérationnel concerné, des dépenses encourues pour la mise en œuvre d'opérations ou de parties d'opérations dans des zones de niveau NUTS 3 jouxtant les zones éligibles pour ce programme qui sont visées à l'article 7 , paragraphe 1 , du règlement (CE) $n^{\circ}$ 1083/2006 ou entourées par de telles zones. Dans des cas exceptionnels convenus entre la Commission et les États membres, cette flexibilité peut être étendue aux zones de niveau NUTS 2 dans lesquelles se situent les zones visées à l'article 7, paragraphe 1, du règlement $(\mathrm{CE}) \mathrm{n}^{\circ} 1083 / 2006 .^{15}$

Un effort de flexibilité a été réalisé également dans les règlements des nouveaux instruments de la Politique de voisinage. L'Instrument d'aide de pré-adhésion (IAP), destiné principalement aux pays candidats officiels, peut être utilisé sur le territoire de pays tiers non-partenaires. Il bénéficiera aussi aux candidats potentiels des Balkans occidentaux pour le volet coopération transfrontalière. L'objectif est de ne pas tuer des 
projets de développement régional en adoptant une définition trop restreinte des zones éligibles à condition que «le projet ou programme concerné (ait) une dimension régionale, transfrontalière, transnationale ou mondiale. $»^{16}$

Dans l'Instrument européen de voisinage et de partenariat (IEVP), on a également prévu une plus grande souplesse géographique, avec en particulier la reprise de la notion de bassin maritime commun, définie dans le règlement général des fonds structurels du 11 juillet 2006. La notion de bassin maritime est même élargie par rapport à ce qui existait auparavant. Ce sont les régions bordières de niveau NUTS 2 qui sont éligibles, ainsi que les régions limitrophes situées sur le territoire de pays tiers :

Les programmes de coopération transfrontalière [...] peuvent couvrir toutes les régions frontalières suivantes : [...] toutes les unités territoriales côtières de niveau NUTS 2 ou équivalent, situées en bordure d'un bassin maritime commun aux États membres et aux pays partenaires.

[...] Pour assurer la poursuite de la coopération existante et dans d'autres cas justifiés, les unités territoriales limitrophes de celles visées au paragraphe 1 peuvent être autorisées à participer aux programmes de coopération transfrontalière dans les conditions fixées dans le(s) document(s) de stratégie spécifique(s) visé(s) à l'article 7 , paragraphe $3 .{ }^{17}$

Au nom du principe de cohérence de l'aide, des pays non mentionnés dans les annexes de l'IEVP et de l'IAP pourront donc bénéficier de ces instruments. ${ }^{18}$

\section{Simplification des règles de financement de la coopération transfrontalière}

La Commission souhaite mettre un terme aux anciennes difficultés d'articulation des différents instruments financiers précédemment utilisés (Interreg, Tacis, Phare...). Dans le cadre d'un programme de coopération transfrontalière qui implique des pays membres et des pays partenaires, ce sont les règles du Feder, définies dans le règlement général des fonds structurels, qui seront appliquées. Les règlements de l'IAP et de l'IEVP sont explicites sur ce point. ${ }^{19}$

31 Dans l'IAP ${ }^{20}$, on évoque même la possibilité pour les pays candidats de gérer les fonds structurels de façon décentralisée pour les aider à se préparer à la future politique de cohésion. Il s'agit d'aider les pays candidats officiels à se préparer à leur adhésion. Outre cela, une telle harmonisation des procédures de financement des projets de développement régional des deux côtés de la frontière externe de l'Union peut renforcer l'efficacité du dispositif en facilitant son financement et sa gestion. ${ }^{21}$

Enfin, outre la confirmation de la dimension conjointe des programmes opérationnels ( $c f$. IEVP, Titre III, article 9), la Commission a instauré une simplification drastique du financement de la coopération transfrontalière et transnationale. Elle a créé un instrument financier unique. Au lieu de financer des projets de coopération transfrontalière ou transnationale avec des instruments financiers différents de part et d'autre de la frontière externe, ce qui posait d'insolubles problèmes d'incompatibilité réglementaire, on n'utilisera plus qu'un instrument. Cette démarche obligera les partenaires à renforcer encore leur coopération et confortera la philosophie d'ensemble des programmes conjoints. ${ }^{22}$ 


\section{Des blocages à venir et des insuffisances demeurent}

33 La politique de cohésion de l'UE fait preuve de souplesse depuis sa création (Leclerc, 2003). La redéfinition régulière de ses objectifs témoigne d'un souci constant d'adaptation. Les règlements mis en œuvre en janvier 2007 n'y échappent pas car ils apportent des évolutions significatives au cadre existant. Néanmoins, il reste fort à faire pour accroitre l'efficacité des instruments financiers à la disposition de la Commission européenne et pour renforcer la pertinence des espaces de coopération définis. On ne fera pas ici une présentation exhaustive de tous les problèmes susceptibles d'émerger à court et à moyen termes. On mettra seulement l'accent sur quelques catégories de difficultés à venir.

\section{Le voisinage est-il bien calibré ?}

34 La notion de voisinage définie par Romano Prodi dans la fameuse communication de $2003^{23}$ est reprise telle quelle dans les règlements les plus récents de la politique européenne de voisinage. Le voisinage est défini de façon très stricte à partir du seul critère de la proximité géographique et de l'existence d'une frontière commune. Les exceptions sont rares. Azerbaïdjan et Arménie profitent de la Politique de voisinage car ils appartiennent à l'ensemble régional du Caucase considéré par la Commission comme un tout. La Jordanie, par exemple, n'a pas non plus de frontière commune avec l'Union et doit à son appartenance à l'ensemble régional méditerranéen. Malgré ces quelques entorses au principe de la proximité géographique, la définition du voisinage peut paraître trop restreinte. Pour créer les conditions d'une plus grande efficacité en matière de cohésion, ne vaudrait-il pas mieux mettre en avant une définition plus fonctionnelle du voisinage? En d'autres termes, prendre également en considération des régions plus éloignées ou des pays tiers avec lesquels il existe des interactions fortes. Ce problème se pose à plusieurs échelles.

L'IEVP par exemple permettra de financer des projets de coopération transfrontalière et transnationale dans des ensembles territoriaux relativement étroits. Il apportera une certaine souplesse dans la délimitation des territoires éligibles, mais davantage du côté communautaire que du côté des pays tiers. Cela peut poser quelques problèmes. On peut par exemple penser que certaines régions des pays baltes entretiennent des relations économiques fortes avec des régions russes situées en dehors des périmètres qui vont profiter du volet « coopération territoriale » de l'IEVP. Elles risquent ainsi de manquer le coche d'un développement économique plus soutenu en n'obtenant pas de précieux financements. La géographie des interactions entre le territoire communautaire et les pays tiers est assez mal connue. Un rapport récent remis à la Commission européenne (Grasland, 2006) montre qu'elles existent, qu'elles varient beaucoup selon les régions européennes et qu'elles impliquent parfois des régions de pays tiers trop éloignées pour bénéficier du volet coopération territoriale. Certains États membres de l'UE ont des relations fortes avec des pays voisins, tandis que d'autres ont des relations plus fortes avec d'autres États plus éloignés. Ce problème a été relevé récemment par la Commission européenne elle-même dans une communication où il est recommandé de tenir compte des interactions qui existent avec des pays qui ne sont pas strictement voisins: "Nous devrions aussi porter notre attention au-delà du voisinage immédiat de l'Union, afin de travailler avec « les voisins de nos voisins » ${ }^{24} \mathrm{En}$ 
bref, les nouveaux outils de la Politique de voisinage apporteront des améliorations pour la coopération à l'échelle locale, mais ils seront insuffisants à une échelle plus petite dans des territoires plus vastes. On pourrait rétorquer qu'il est vain de vouloir étendre à l'infini l'éligibilité des territoires pris en compte par des instruments comme l'IEVP et que la Politique de voisinage n'a pas vocation à s'étendre à l'infini aux voisins des voisins des voisins. Néanmoins, on peut craindre l'émergence de blocages.

L'IAP risque aussi de poser quelques problèmes car sa flexibilité n'est pas si grande qu'on pouvait l'espérer. Il s'adresse en théorie aux pays candidats potentiels des Balkans occidentaux, mais seulement pour certains volets. L'article 20 du préambule indique que ces pays devraient pouvoir bénéficier de la totalité des volets. Mais les modalités restent floues car ils ne sont pas habilités à la gestion décentralisée des crédits européens. Par ailleurs, l'article 10 indique que le volet "développement régional " ne pourra profiter qu'aux pays candidats officiels. Cela risque de nuire à l'amélioration de la coopération à l'échelle régionale entre pays membres, candidats officiels et candidats potentiels.

Les volets « développement régional », « développement des ressources humaines » et «développement rural» ne devraient être accessibles qu'aux seuls pays candidats accrédités pour gérer des fonds d'une manière décentralisée, afin de les aider à se préparer à la période suivant l'adhésion, et notamment à la mise en œuvre des politiques de cohésion et de développement rural de la Communauté.

Les pays candidats potentiels et les pays candidats qui n'ont pas été accrédités pour gérer des fonds d'une manière décentralisée devraient toutefois pouvoir bénéficier, au titre du volet «aide à la transition et renforcement des institutions ", de mesures et d'actions de nature similaire à celles qui seront disponibles au titre des volets « développement régional », « développement des ressources humaines » et « développement rural ». ${ }^{25}$

On appréciera le flou de la formulation et surtout l'usage qui est fait du conditionnel. Autrement dit, la Commission et le Conseil européen tentent d'effacer, par un tour de passe rhétorique, la restriction posée dans la première partie de l'article. Dans ce cas, pourquoi avoir instauré une restriction? Cela présage de problèmes dans la mise en œuvre locale de cette disposition.

Enfin, on a dit plus haut que le règlement Feder doit être suivi dès qu'il s'agit de coopération transfrontalière des deux côtés de la frontière externe. En réalité, cette simplification ne profite qu'au volet transfrontalier et pas au transnational. Cette disposition est d'autant plus difficile à comprendre que l'IAP permet de mettre en œuvre des projets de coopération à l'échelle transnationale. ${ }^{26}$

\section{Un problème de coordination institutionnelle entre outils et entre administrations}

39 L'IEVP et l'IAP sont des instruments financiers d'aide à des pays tiers. À ce titre, ils doivent être gérés par la DG Relex du Commissariat pour les Relations extérieures. Or on peut s'interroger sur les compétences de la DG Relex en matière de développement régional et d'aménagement du territoire. La question se pose avec d'autant plus de force qu'il s'agira de financer des projets de développement sur le territoire de pays étrangers, ce qui semble a priori encore plus complexe que dans le territoire communautaire. Par ailleurs, la DG Relex sera-t-elle amenée à gérer les fonds Feder dépensés sur le territoire de pays tiers? 
40 À ces problèmes potentiels s'ajoute celui de la coordination entre les diverses administrations concernées au sein de la Commission. La mise en œuvre de l'IAP et de l'IEVP est en théorie du ressort de la DG Relex. Mais dans le cas de financement de projets de coopérations transfrontaliers et transnationaux, quelle administration aura le dernier mot? Est-ce la DG Regio, pour les unités territoriales communautaires éligibles? Est-ce la DG Relex pour les territoires éligibles situés dans les pays tiers? Le problème ne devrait pas émerger le long des frontières orientales avec les pays de la CEI, où des structures conjointes existent déjà. Mais rien n'est moins sûr dans les Balkans occidentaux où de telles structures n'existent pas et ne sont pas prévues par l'IAP. Un comité IAP est bien institué par l'article 14 du règlement mais il n'est composé que par des représentants des États membres.

41 Pour être totalement efficaces, il faudrait aussi que les divers instruments mis en œuvre soient compatibles. Le problème semble résolu en ce qui concerne les procédures financières, puisque c'est le règlement du Feder qui sera utilisé de plus en plus des deux côtés de la frontière communautaire. Mais il faudra aussi que les objectifs énoncés dans les documents qui servent de base aux relations contractuelles entre l'UE et ses voisins (plans d'action et accords de stabilisation et d'association) soient compatibles avec les projets de coopération transfrontaliers et transnationaux financés. De même, il faudrait que les grands objectifs de l'IEVP et de l'IPA en terme de développement régional et de coopération territoriale soient compatibles avec les projets de développement des régions périphériques de l'Union pour que celles-ci profitent au maximum de la politique de coopération avec les pays tiers proches. Un projet de coopération transfrontalière et transnationale aura d'autant plus de chance d'avoir un impact positif que les objectifs visés sont compatibles avec les projets portés par la DG Relex dans les relations avec les voisins.

Des exemples le montrent le long de la frontière avec l'ex-URSS. La Russie est très engagée dans une coopération territoriale avec la Finlande à l'échelle locale et à l'échelle régionale (Marin, 2005). Elle a signé des accords entre autres pour la construction d'infrastructures de transports communes qui permettront de relier Saint-Pétersbourg, et donc Moscou, à Helsinki. Pendant ce temps, les autorités estoniennes ne parviennent pas à s'entendre avec leurs homologues russes pour l'augmentation du gabarit du pont qui traverse la rivière Narva entre les deux pays. Le passage de la frontière est ici un goulet d'étranglement qui gène les relations commerciales et pèse négativement sur le développement économique de la région estonienne de Narva, dont certaines industries pourraient profiter d'une ouverture du marché russe. C'est typiquement un problème qui ne peut être résolu efficacement que par le biais d'une coopération totale entre DG Relex, qui assure l'avancement de certains dossiers avec les États proches, et la DG Regio, qui est en charge du développement local et qui veille à l'intérêt de toutes les régions européennes.

On est loin de cette situation aujourd'hui et la Russie joue facilement les États européens les uns contre les autres pour avancer ses propres projets, au risque de court-circuiter les États baltes qui souhaiteraient pourtant jouer un rôle important d'interface entre elle et l'Europe communautaire. Dans ce cas, l'instauration de comités conjoints de coopération, dans le cadre de l'IEVP, n'est pas suffisante puisque ces comités auront un rôle souvent plus technique que politique. L'avancement de la coopération, profitable aux régions européennes périphériques, ne peut passer que par 
une action politique du Commissariat aux Relations extérieures à un niveau plus élevé, conjuguée à celle des comités techniques.

La Commission exprime régulièrement sa volonté de mieux coordonner les différentes politiques et les différents instruments mis en œuvre pour la coopération avec des pays tiers. Pour autant, il y a loin de la coupe aux lèvres. La lecture des documents stratégiques et des plans d'action signés avec les pays voisins orientaux de l'UE le confirme. Les paragraphes qui renvoient explicitement à la coopération territoriale et au développement régional y tiennent une place mineure pour ne pas dire moins. Le cas de l'Ukraine est exemplaire. Le document stratégique annonce la couleur en mettant l'accent sur la nécessité de bien combiner les différentes politiques de l'Union vis-à-vis de ce pays :

Les relations avec l'Ukraine sont affectées par plusieurs autres politiques communautaires, ce qui explique pourquoi il est si important de sélectionner la bonne «combinaison de mesures». Ceci concerne, en particulier, la politique étrangère et de sécurité commune (PESC), la gestion des frontières et la migration, le commerce, l'énergie, les transports et la protection de l'environnement. Les aspects liés à la technologie de l'information et de la communication sont également très importants. $C f$. description détaillée des aspects de la " combinaison de mesures » en annexe $5 .{ }^{27}$

Further elaborate a concept for a national sustainable transport policy for the development of all transport modes, coherent with the EU's White Paper on transport. Develop an infrastructure policy in order to identify and evaluate the priority infrastructure projects in various sectors and continue participation in the joint development of the Pan-European Corridors and Areas as well as in the TRACECA programme..$^{28}$

Mais dans le détail du plan d'action, qui est censé poser les priorités politiques de la relation entre l'UE et l'Ukraine, les aspects relatifs à la coopération territoriale et au développement régional disparaissent. On souligne l'impact de certaines politiques communautaires sur les relations avec l'Ukraine, mais on fait l'impasse sur l'impact des évolutions sociales et économiques de l'Ukraine sur les régions européennes. Dans le Plan d'action de la Moldavie, le problème se pose aussi. L'idée de développement régional apparaît dans les nouvelles perspectives du partenariat mais pas dans la liste des priorités développées en préambule. L'objectif de coopération renvoie seulement à l'idée de coopération internationale pour la gestion des flux migratoires et pour la surveillance des frontières (question de sécurité).

Autre problème, il semble que la Commission ait décidé d'imposer à nouveau aux pays membres l'utilisation du guide pratique des procédures contractuelles pour la mise en œuvre des programmes de coopération extérieurs. Ce document concerne au premier chef la coopération territoriale aux frontières externes de l'Union. ${ }^{29}$ Or, certaines de ces recommandations sont incompatibles avec la réglementation du nouvel instrument de voisinage et de partenariat. ${ }^{30}$

\section{Quelques problèmes persistants encore dans le mode de financement des projets conjoints}

Le financement des projets de coopération a été simplifié mais certains problèmes, pourtant soulevés par des États membres lors des réflexions préparatoires à la rédaction des nouveaux règlements, n'ont pas été réellement résolus. L'un de ces problèmes est celui de la responsabilité des États membres. Les termes utilisés dans le règlement général des fonds structurels laissent entendre qu'ils sont responsables de la 
façon dont les fonds seront dépensés. En soi, cela est normal. Mais peut-on être aussi tranché lorsqu'il s'agit de coopération territoriale avec des pays tiers? Les autorités estoniennes, par exemple, s'en sont alarmées, indiquant qu'elles se refusaient à répondre de la manière dont les fonds IEVP seront dépensés en Russie par exemple. ${ }^{31} \mathrm{Le}$ règlement de l'IEVP apporte des éléments de réponse sans trancher dans le sens attendu. La Commission se réserve le droit de mener des actions d'audit et de contrôle, mais ce sont les États membres qui sont chargés de veiller à la légalité de toutes les opérations réalisées. Outre les réticences formulées par les États membres sur le principe même de responsabilité financière, on peut s'interroger sur la bonne volonté des autorités russes dans ce domaine : accepteront-elles que des audits et des contrôles soient faits par des fonctionnaires européens sur leur territoire ? ? $^{32}$

Autre problème, on ne sait pas avec certitude si le système de financement pluriannuel des projets de coopération sera modifié. Actuellement, ce système est responsable de retards dans le financement de projets de coopération du côté des pays partenaires non-membres. Pour recevoir leurs fonds Tacis par exemple, les pays bénéficiaires étaient jusqu'à présent obligés de signer chaque année des conventions de financement. C'est une façon pour l'UE de sauvegarder ses intérêts financiers, mais elle ralentit l'attribution des fonds..$^{33}$

Ce problème est très sensible pour les autorités des États membres car l'instrument financier unique de financement de la coopération territoriale les rend solidaire des États voisins, quelles que soient la qualité de leurs relations politiques. Le ministère estonien du développement régional a adressé une question simple aux Commissaires européens. Que se passe-t-il si le pays partenaire, en l'occurrence la Russie, ne signe pas dans les temps les conventions de financement qui lui permettent de recevoir ses fonds IEVP ? Est-ce que le versement est bloqué également pour les régions frontalières situées sur le territoire communautaire? La question ne se posait pas en ces termes avant 2007 car les crédits qui finançaient la coopération venaient de budgets différents (Interreg III A et Tacis CTF par exemple). Mais des problèmes existaient déjà. La Russie a signé à plusieurs reprises avec retard les conventions de financement qui lui permettaient de recevoir ses fonds Tacis : par exemple, la signature pour les fonds au titre de l'année 2004 n'est intervenue qu'en 2005. Entre-temps, les partenaires russes impliqués dans des projets de coopérations retenus devaient les financer sur leurs fonds propres, très limités, tandis que l'argent Feder était déjà dépensé sur le versant côté communautaire de la frontière. De ce fait, certains projets ont accusé des retards. Il semble que la Commission penche pour le versement d'une dotation globale IEVP au lancement de chaque projet. Ce serait une réelle amélioration.

\section{L'insuffisance chronique des moyens budgétaires}

50 L'UE et ses politiques ne doivent certes pas se substituer à l'ensemble des acteurs locaux et nationaux du développement régional. Mais elle devrait proposer des enveloppes financières plus incitatives pour servir de levier aux relations transfrontalières et transnationales avec les voisins. La baisse du budget européen ne va pas dans le bon sens.

51 Le budget de la politique de cohésion est en augmentation par rapport à 2000-2006, mais la lecture des règlements montre que le volet " coopération territoriale " est le moins bien doté de tous avec une enveloppe de 7,75 milliards d'euros pour la période 
2007-2013. C'est la coopération transfrontalière qui reçoit la plus grande part des crédits affectés à ce volet (73,86 \%). Ces chiffres sont à mettre en regard de l'importance des régions frontalières dans l'espace communautaire : 181 millions d'habitants, soit plus de $37 \%$ de la population communautaire. Alors que le volet coopération transnationale, dont les objectifs sont pourtant complémentaires, en reçoit beaucoup moins $(20,95 \%){ }^{34}$ Sans compter que ces 7,75 milliards d'euros seront attribués à l'ensemble des régions frontalières et pas seulement à celles qui se trouvent le long des frontières externes de l'UE. La somme attribuée à ces dernières sera donc faible. La contribution du Feder aux programmes transfrontaliers et aux bassins maritimes au titre de l'IAP et de l'IEVP ne s'élève qu'à un peu moins de 814 millions d'euros, auxquels il faut ajouter une somme identique prélevée sur l'IEVP et l'IAP. ${ }^{35}$ C'est trop peu pour espérer des résultats sensibles, mais la Commission fait face à un principe de réalité plus fort que tout: les États membres n'ont pas souhaité augmenter substantiellement le budget communautaire. Difficile de dégager des marges de manœuvres dans ce contexte.

Graphique 1. Part des voisins de l'UE 27 dans l'aide au développement versée par la Commission.

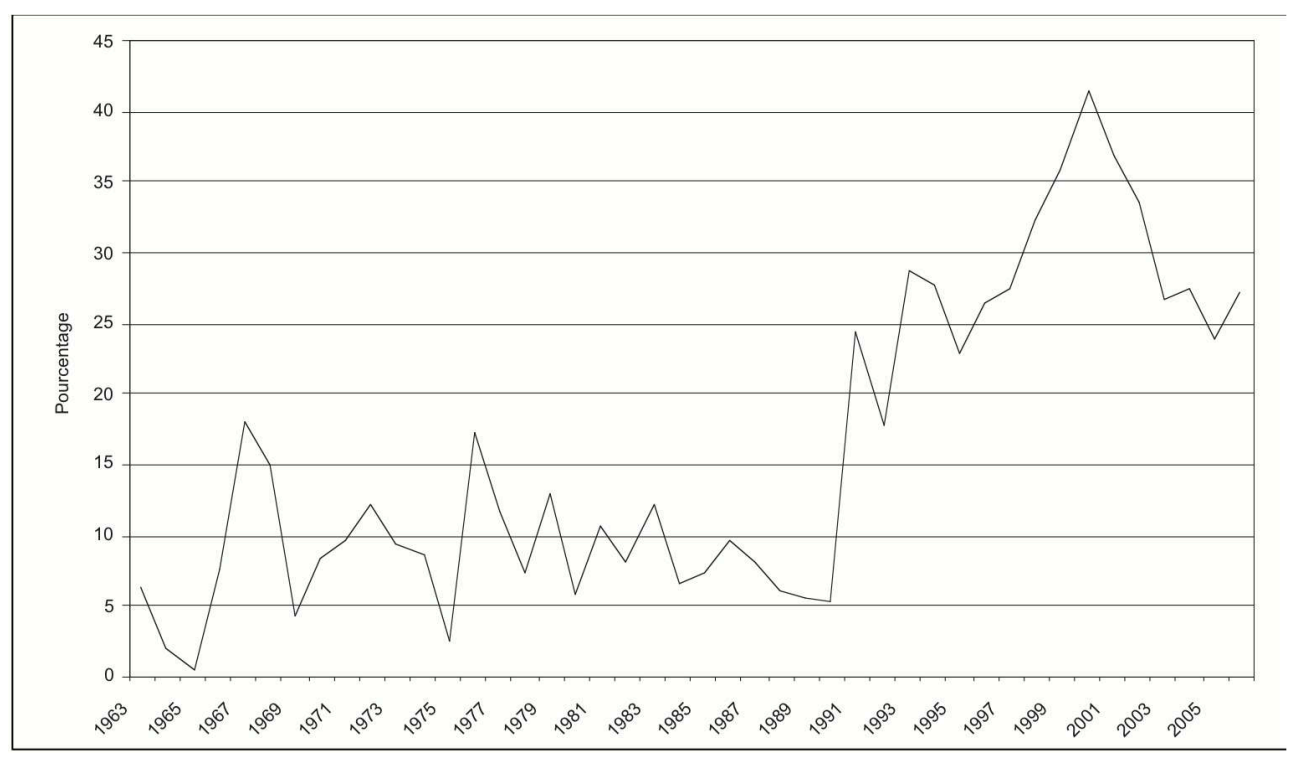

Cette pénurie budgétaire s'inscrit dans un processus continu de recul entamé il y a déjà longtemps. Les pays qui forment actuellement le voisinage géographique, et non institutionnel, de l'UE à 27 recevaient dans les années 1960 plus de $37 \%$ de l'aide au développement totale versée par l'UE (Commission + États membres de l'UE 15 ; graphique 3). L'essentiel de cette aide allait aux pays en développement riverains de la Méditerranée. Actuellement, les pays du voisinage géographique ne reçoivent plus que $10,4 \%$ de cette aide. Si l'on décompose celle-ci, on constate que la Commission européenne, qui gère les fonds de la Politique de voisinage et de la coopération territoriale, a augmenté ses déboursements en valeur entre les années 1960 et 2000 (graphique 1). Toutefois, l'aide totale au développement que verse la Commission dans l'ensemble des pays en développement augmente plus vite que celle adressée au pays $\mathrm{du}$ voisinage géographique (graphique 2). De plus, les pays membres ont réduit leur aide bilatérale, dont la valeur est très supérieure à celle versée par la Commission. Elle serait pourtant bien venue dans les régions frontalières des pays voisins. Celles-ci sont souvent pauvres et dépourvues des ressources budgétaires nécessaires pour participer 
au cofinancement des programmes de coopération territoriale. Les sommes versées par la Commission ne financent jamais l'intégralité des programmes de coopération. Les régions bénéficiaires doivent mettre la main à la poche.

Graphique 2. L'aide au développement versée par la Commission européenne aux pays de son voisinage géographique.

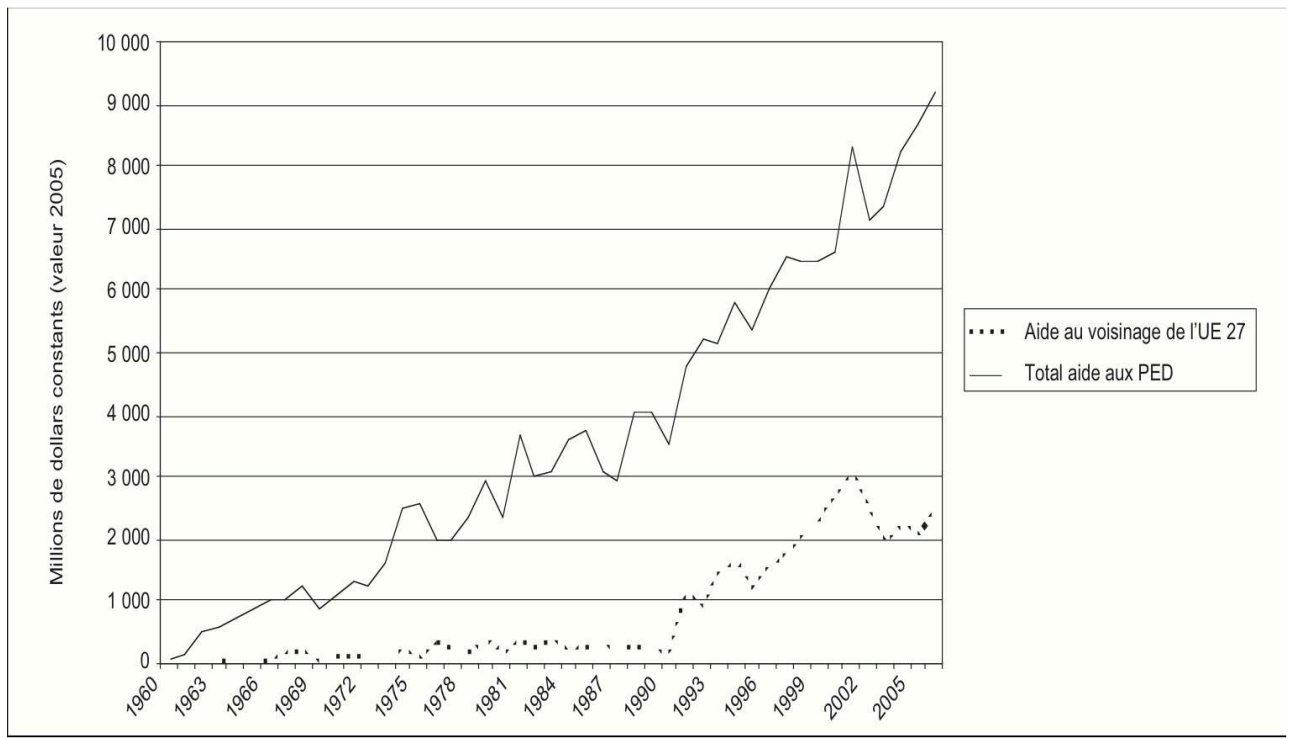

Graphique 3. Part du voisinage et des pays méditerranéens en développement dans l'aide au développement totale de l'UE (pays membres + Commission).

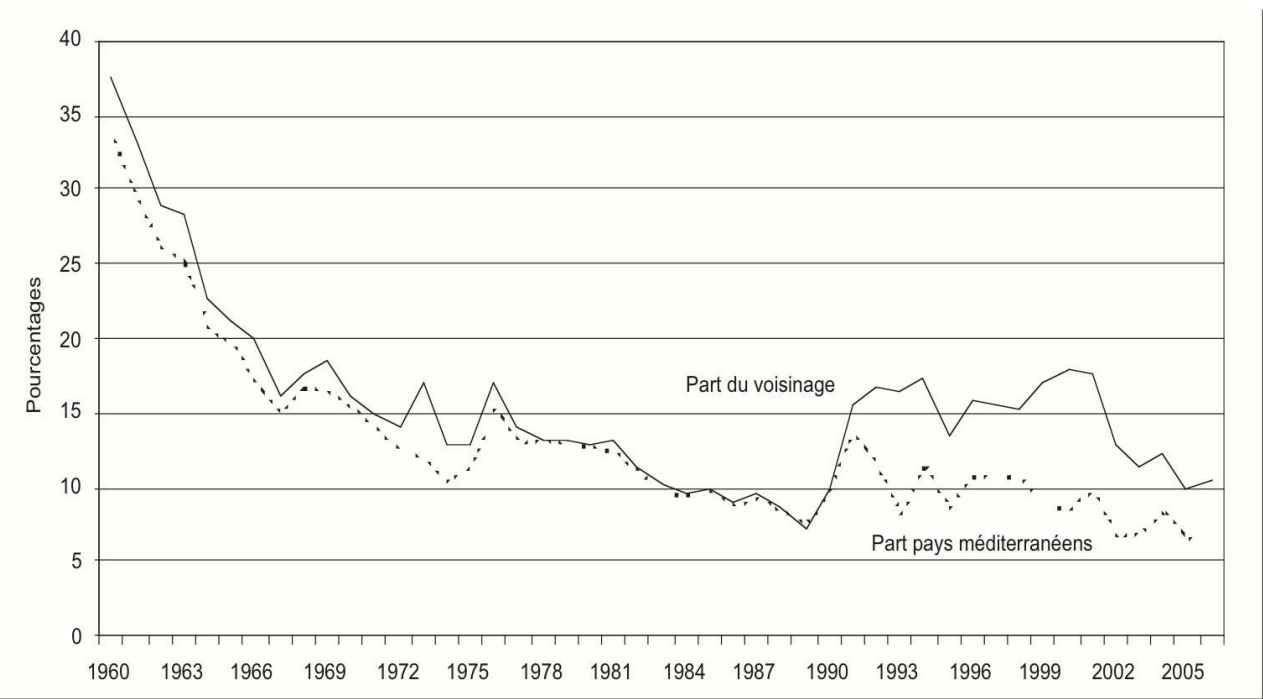

\section{La multiplicité des statuts des pays voisins n'est-elle pas une entrave à la coopération territoriale aux frontières externes ?}

La variété des statuts des pays voisins au regard de leurs relations contractuelles avec l'UE est une gêne et complexifie la coopération à toutes les échelles, car les réglementations ne sont pas les mêmes pour tous les pays : pays candidats officiels, pays candidats potentiels, pays de la PEV, pays ASA, Russie, pays sans relations contractuelles officielles (Belarus). Les textes réglementaires tentent de répondre par 
anticipation à ce problème en rappelant que ce sont les règles financières du règlement général qui s'appliqueront. La Commission a fait un grand pas dans le sens d'une plus grande compatibilité entre instruments. Toutefois, il reste du chemin à faire et on demande à voir l'efficacité des dispositifs proposés. Sans compter que les pays voisins n'ont pas toujours de bonnes relations. Rappelons que les pays de l'annexe II de l'IPA n'auront pas accès au volet coopération transnationale de cet instrument. La Commission européenne tente de simplifier les outils de sa politique extérieure en réduisant leur nombre. Mais les textes contractuels signés par les voisins présentent des différences encore substantielles, ce qui cause un émiettement des voisinages.

Par ailleurs, la volonté affichée par l'UE de stimuler de nouvelles formes de coopération à l'échelle régionale (internationale ou transnationale) ${ }^{36}$ n'est pas encore suivie d'effets. À ce jour, c'est essentiellement par des traités bilatéraux que l'UE entretient des relations contractuelles avec ses voisins. La dimension régionale existe puisque le Commissariat en charge des Relations extérieures divise le voisinage régional de l'UE en grands ensembles, mais ce découpage n'est pas clair et demeure essentiellement nominal. En réalité deux découpages se télescopent. Un en fonction des outils de coopération mis en œuvre : les pays bénéficiant de l'IAP et ceux bénéficiant de l'IEVP. Un en fonction des types de documents contractuels signés avec les pays voisins : ASA et Plans d'actions, mais aussi Accords de Partenariat et de Coopération... En outre, cette dimension régionale est en trompe l'œil car la Commission n'a à ce jour jamais signé de documents juridiquement contraignants (tel un plan d'action) avec des blocs d'États contigus formant des ensembles régionaux. C'est donc une approche bilatérale qui prévaut aujourd'hui dans les relations extérieures de l'UE. Elle n'est pas mauvaise en soi. Elle est même nécessaire. Mais elle n'est pas suffisante. Elle révèle une incapacité à traiter certains problèmes qui ne peuvent être résolus qu'à une échelle régionale. $\mathrm{La}$ coopération territoriale pourrait y aider.

\section{Conclusion}

Les orientations réglementaires montrent de réelles améliorations des dispositifs de coopération territoriale. Ceux-ci allient une plus grande souplesse d'utilisation des crédits du Feder à l'extérieur de l'UE et une définition des zones éligibles plus large. Elles peuvent augurer d'une meilleure prise en compte des interactions sociales et économiques qui existent entre le territoire communautaire et les pays voisins. En procédant ainsi, l'UE applique à ses frontières externes l'objectif schumanien d'ouverture des frontières internes (Foucher, 2007). Il n'empêche que l'UE continue de penser sa politique de cohésion dans un cadre territorial fermé. Aussi, certains blocages sont encore susceptibles d'entraver l'efficacité des outils destinés au développement régional à l'échelon national et régional.

Des efforts importants ont été accomplis pour prendre en considération les interactions souvent anciennes qui existent entre les territoires communautaires ou non situés le long des frontières externes de l'UE, c'est-à-dire à l'échelle locale. En revanche, les nouvelles réglementations ne prennent toujours pas assez en considération les interactions à plus petite échelle, c'est-à-dire entre des régions adjacentes qui constituent des territoires transnationaux plus vastes que les territoires transfrontaliers. Ces problèmes mettent en relief la difficulté de coordonner, d'une part, les actions menées par la DG Regio et la DG Relex et, d'autre part, d'assurer une 
cohérence entre les différents instruments mis en œuvre dans le cadre de la coopération avec les pays tiers.

Les évolutions récentes et les blocages constatés ou à venir montrent que l'UE peine encore à concevoir un nouveau type de frontière externe, malgré la mise en œuvre des programmes de voisinage. Néanmoins, les tâtonnements de la Commission prouvent qu'une frontière multiforme est en train d'apparaître, mêlant fermeture et ouverture, coopération locale et transnationale, approche bilatérale et régionale. C'est sans doute le modèle vers lequel l'Union, les États membres et les pays voisins s'acheminent : une frontière souple à géométrie variable. Pour continuer d'avancer dans la direction indiquée par la Commission, il faudra poursuivre la réforme des règlements de la coopération territoriale, progresser vers une plus grande coordination intersectorielle et surtout mettre davantage d'argent sur la table. Un contexte nouveau est susceptible de favoriser cette orientation avec l'affirmation institutionnelle de la "cohésion territoriale ». Derrière cet objectif, mentionné explicitement dans le Traité de Lisbonne (article 2), c'est la solidité des liens de proximité géographique (Vignon, 2000) et une meilleure coordination des politiques sectorielles ayant un impact spatial qui sont désormais reconnues.

\section{BIBLIOGRAPHIE}

Albrechts L., Healey P., Kunzmann K., 2003, « Strategic Spatial Planning and Regional Governance in Europe ", APA Journal, vol. 69, 2.

Allison G., Zelikow P., 1999, Essence of Decision, Explaining the Cuba Missile Crisis, New York, Longman.

Beckouche P., Richard Y., 2005, Atlas d'une nouvelle Europe, Paris, Autrement, $2{ }^{\mathrm{e}}$ édition augmentée et mise à jour.

Beckouche P., Grasland C., Didelon C., Richard Y., 2008, Europe in the World. Territorial Evidence and Visions, Bruxelles, Bietlot.

Benz A., 2002, « How to Reduce the Burden of Coordination in European Spatial Planning », dans Faludi, A. (ed.), European Spatial Planning, Lincoln Institute of Land Policy, Cambridge,

p. $139-158$.

Böhme K., Schön P., 2006, « From Leipzig to Leipzig », disP, 2.

Camagni R., 2006, « Compétitivité territoriale : la recherche d'avantages absolus », Reflets et Perspectives, XLV,1.

Christiansen T., Jorgensen K. E., 2000, « Transnational Governance "Above" and "Below" the State : The Changing Nature of Borders in the New Europe », Regional \& Federal Studies, vol. 10, 2.

Commission européenne, 1996, Premier rapport sur la cohésion économique et sociale, Luxembourg, Luxembourg, Office des publications officielles des Communautés européennes. 
Commission européenne, 1999, SDEC, Schéma de développement de l'espace communautaire : vers un développement spatial équilibré et durable du territoire de l'Union européenne, Luxembourg, Office des publications officielles des Communautés européennes.

Commission européenne, 2001, Unité de l'Europe, solidarité des peuples, diversité des territoires, Deuxième rapport sur la cohésion économique et sociale, Luxembourg, Office des publications officielles des Communautés européennes.

Commission européenne, Livre blanc sur la gouvernance, Communication COM (2001) 428 final du 25 juillet 2001.

Commission européenne, 2004, Un nouveau partenariat pour la cohésion-Convergence, compétitivité, coopération, Troisième Rapport sur la cohésion économique et sociale, Luxembourg, Office des publications officielles des Communautés européennes.

Commission européenne, 2005, Des réseaux pour la paix et le développement, Extension des grands axes transeuropéens de transport vers les pays et régions voisins, Rapport de Groupe à Haut Niveau présidé par Loyola de Palacio.

Commission européenne, 2007, Extension des grands axes transeuropéens de transports aux pays voisins, Lignes directrices concernant les transports en Europe et dans les pays voisins, COM (2007) 32 final.

Commission européenne, 2007, Des régions en pleine croissance, une Europe en pleine croissance, Quatrième rapport sur le cohésion économique et sociale, Luxembourg, Office des publications officielles des Communautés européennes.

Davezies L., 2002, « Notes de lecture du deuxième rapport sur la cohésion : unité de l'Europe, solidarité des peuples, diversité des territoires », Territoires $2020, \mathrm{n}^{\circ} 5$.

Didelon C., Grasland C. (dir.), 2006, Europe In The World, 3 volumes. Rapport adressé à la Commission des Communautés européenne, dans le cadre du Programme Orate en novembre 2006. Version finale du rapport disponible en ligne sur le site internet du Programme Orate (www.espon.eu).

Faludi A., Waterhout B., 2002, The making of the European spatial development perspective : no masterplan, London, New York, Routledge.

Faludi A., Waterhout B., 2005, « The usual suspects : the Rotterdam EU informal ministerial meeting on territorial cohesion », Tijdschrift voor Economische en Sociale Geografie, vol. 96, $\mathrm{n}^{\circ} 3$.

Foucher M., 1991, Fronts et frontières. Un tour du monde géopolitique, Paris, Fayard (2 édition).

Foucher M., 2007, L'obsession des frontières, Paris, Perrin.

Gaulier G., Freudenberg M., Ünal-Kensenci D.,1998, La régionalisation du commerce international : une évaluation par les intensités relatives bilatérales, CEPII, Working Paper $\mathrm{n}^{\circ}$ 98-05.

Gestel T. van, Faludi A., 2005, « Towards a European Territorial Cohesion Assessment Network », Town Planning Review, 76 (1).

Healey P., Khakee P., Motte A. and Needham B., 1997, Making Strategic Spatial Plans : Innovation in Europe, London, UCL Press.

Healey P., 2004, « The Treatment of Space and Place in the New Strategic Spatial Planning in Europe », International Journal of Urban and Regional Research, 28, 1.

Leclerc S., 2003, L'Europe et les régions, Bruxelles, Bruylant. 
Leclerc S., 2003, «L'évolution du cadre juridique et conceptuel de la cohésion économique et sociale : la nouvelle réglementation des fonds structurels (2000-2006)», dans L'Europe et les régions, Bruxelles, Bruylant, pp. 71-92.

Marin A., 2005, « Du bon voisinage en relations internationales. La frontière finno-russe, laboratoire et modèle de coopération en Europe ", Revue d'Études Comparatives Est-Ouest, vol. 36, n 3.

O'Dowd L., 2002, « The Changing Significance of European Borders ", Regional \& Federal Studies, vol. $12, \mathrm{n}^{\circ} 4$.

Paasi A., 1998, « Boudaries as Social Processes : Territoriality in the World of Flows », Geopolitics, vol. 3,1 .

Perkmann M., 1999, « Building governance across structures across European Borders », Regional Studies, 33.

Rees J.-H., Deblanc C., 2004, « La politique communautaire des réseaux transeuropéens de transport et l'aménagement du territoire en Europe », Territoires 2020, 11.

Richardson T., Jensen O.B., 2003, « Linking Discourse and Space : Towards a Cultural Sociology of Space in Analysing Spatial Policy Discourses », Urban Studies, vol. 40, 1.

Simon H., 1957, Models of Man: Social and Rational, New York, John Wiley.

Vignon J., 2000, «Cohésion sociale... et territoriale ? ", Agir. Revue générale de stratégie, 5.

\section{NOTES}

1. Premier rapport sur la cohésion, p. 109.

2. Deuxième rapport sur la cohésion, p. 4.

3. Troisième rapport sur la cohésion, p. 157.

4. Quatrième rapport sur la cohésion, p. XIV.

5. SDEC, p. 39.

6. Voir: http://ec.europa.eu/ten/transport/external_dimension/index_fr.htm (consulté le 15/10/07).

7. Décision du Conseil relative aux orientations stratégiques communautaires en matière de cohésion, 18 août 2006, p. 49.

8. Document L'État et les perspectives du territoire de l'Union européenne, 2007, page 22.

9. Agenda territorial de l'Union européenne. Vers une Europe plus compétitive et durable avec des régions diverses, Accepté à l'occasion de la réunion informelle des ministres du Développement urbain et de la Cohésion territoriale, le 24/25 mai 2007 à Leipzig, p. 8.

10. Cour des comtes, Rapport spécial $n^{\circ} 11 / 2001$, relatif au programme Tacis de coopération transfrontalière, accompagné des réponses de la Commission, Joce, 23/11/2001.

11. Communication de la Commission aux États membres, du 28 avril 2000, fixant des orientations pour une initiative communautaire concernant la coopération transeuropéenne et destinée à favoriser un développement harmonieux et équilibré du territoire européen - Interreg III, Joce, Objectifs généraux, alinéa 5, 23/05/2000.

12. Cf. Manuel pour la Coordination du Financement Tacis et Interreg, p. 11 et 12.

13. Communications de la Commission aux Conseil et au Parlement européen, «Jeter les bases d'un nouvel instrument de voisinage", Bruxelles, le 1.7.2003 COM (2003) 393 final et "L'Europe élargie - Voisinage : un nouveau cadre pour nos relations avec les voisins de l'Est et du Sud", COM (2003) 104 final. 
14. Règlement (CE) No 1080/2006 du Parlement européen et du Conseil, du 5 juillet 2006, relatif au Fonds européen de Développement régional et abrogeant le règlement (CE) n ${ }^{\circ}$ 1783/1999. Voir aussi le chapitre 3 , article 21 , $§ 3$.

15. Idem, chapitre 3 , article $21, \S 1$.

16. Règlement (CE) No 1085/2006 du Conseil, du 17 juillet 2006, établissant un instrument d'aide de préadhésion (IAP), titre IV, article 24, 31/07/2006. Voir aussi titre II, article 9, § 1, Joce, $31 / 07 / 2006$. Les pays de l'annexe I sont les candidats officiels. Ceux de l'annexe II sont les candidats potentiels.

17. Règlement (CE) $\mathrm{N}^{\circ} 1638 / 2006$ du Parlement européen et du Conseil du 24 octobre 2006 arrêtant des dispositions générales instituant un instrument européen de voisinage et de partenariat, Titre III, article 8, alinéas 1 et 2 , Joce, 9/11/2006. Voir aussi Titre III, article 9 , § 5 .

18. Règlement (CE) $\mathrm{N}^{\circ} 1085 / 2006$ du Conseil, du 17 juillet 2006, établissant un instrument d'aide de préadhésion (IAP), remarques préliminaires, alinéas 29, JOCE, 31/07/2006.

19. Règlement(CE) $\mathrm{N}^{\circ} 1085 / 2006$ du Conseil, du 17 juillet 2006, établissant un instrument d'aide de préadhésion (IAP), Titre II, article 8, alinéa 3, Joce, 31/07/2006. Voir aussi le Règlement (CE) No 1638/2006 du Parlement européen et du Conseil, du 24 octobre 2006, arrêtant des dispositions générales instituant un instrument européen de voisinage et de partenariat, Titre II, article 9 , alinéa 3, Joce, 9/11/2006.

20. Voir notamment l'alinéa 19 du préambule et le Titre II, article 10, alinéa 1.

21. Règlement (CE) No 1085/2006 du Conseil, du 17 juillet 2006, établissant un instrument d'aide de préadhésion (IAP), remarques préliminaires, $§ 14$ et 15, Joce, 31/07/2006.

22. Règlement (CE) No 1085/2006 du Conseil, du 17 juillet 2006, établissant un instrument d'aide de préadhésion (IAP), Titre II, article 9, § 3, Joce, 31/07/2006.

23. L'Europe élargie - Voisinage : un nouveau cadre pour nos relations avec les voisins de l'Est et du Sud, Communication de la Commission aux Conseil et au Parlement européen, COM (2003) 104 final.

24. Communication de la Commission au Conseil et au Parlement européen relative au renforcement de la Politique européenne de Voisinage, COM (2006) 726 final, 4/12/2006.

25. Règlement (CE) N $\mathrm{N}^{\mathrm{O}}$ 1085/2006 du Conseil, du 17 juillet 2006, établissant un instrument d'aide de préadhésion (IAP), Préambule, $§ 19$ et 20.

26. Règlement (CE) N $\mathrm{N}^{\circ} 1085 / 2006$ du Conseil, du 17 juillet 2006, établissant un instrument d'aide de préadhésion (IAP), Titre II, Article $9, \S 3$.

27. IEVP - Ukraine. Document de Stratégie 2007-2013, p. 15.

28. Plan d'action Union européenne - Ukraine.

29. Guide pratique des procédures contractuelles dans le cadre des actions extérieures, 2007, Commission des Communautés européennes, européen (http://ec.europa.eu/europeaid/ work/ procedures/implementation/practical_guide/index_en.htm).

30. Par exemple, les dispositions relatives au versement de subventions à des organismes à but non commercial (article 6.1.1 p. 99). Cette disposition contredit ce qui est dit dans la réglementation de l'IEVP. Autre exemple, il y a une contradiction entre les articles 13 et 15 de l'IEVP sur la procédure d'évaluation des projets de coopération et le point 6.4.8.6 du guide pratique p. 117. Merci à Madame Margarita Jefimova du ministère de l'Intérieur d'Estonie pour son aide sur ce point.

31. Lettre du ministre du Développement régional de la république d'Estonie, datée du 7 janvier 2005, adressée à Mmes Hübner et Ferrero-Waldner. Estonian proposals on implementing crossborder co-operation at the EU external border under the European Neighbourhood and Partnership Instrument (ENPI).

32. Règlement (CE) No 1083/2006 du Conseil, du 11 juillet 2006, portant dispositions générales sur le Fonds européen de développement régional, le Fonds social européen et le Fonds de cohésion, 
et abrogeant le règlement $(\mathrm{CE}) \mathrm{n}^{\circ} 1260 / 1999$, Titre VI, Chapitre 5 , section I, Article 70 , $\S 1$ et 2 , Joce 31/07/2006.

33. Règlement (CE) $\mathrm{N}^{\circ} 1638 / 2006$ du Parlement européen et du Conseil, du 24 octobre 2006, arrêtant des dispositions générales instituant un instrument européen de voisinage et de partenariat, Titre IV, article $19, \S 1,2$ et 3, Joce 9/11/2006.

34. Règlement (CE) No $1083 / 2006$ du Conseil du 11 juillet 2006, portant dispositions générales sur le Fonds européen de développement régional, le Fonds social européen et le Fonds de cohésion, et abrogeant le règlement (CE) $n^{\circ} 1260 / 1999$, Chapitre 5, Article $21, \S 1$.

35. En réalité, la somme totale ne sera pas de 813 millions d'euros multipliée par deux car les sommes prises sur l'IPA et l'IEVP seront relativement faibles : moins de 466 millions d'euros pour l'IEVP et moins de 244 millions d'euros pour l'IPA. Voir le Règlement (CE) $N^{\circ}$ 1083/2006 du Conseil du 11 juillet 2006, Chapitre 5, Article 21, § 2 et $\S 3$.

36. Lire à ce sujet la Communication de la Commission au Conseil et Parlement européen, relative au renforcement de la politique européenne de voisinage, Bruxelles, le 4.12.2006, COM (2006) 726 final.

\section{RÉSUMÉS}

L'Union européenne continue de se percevoir comme une entité fermée dans de nombreux domaines. Dans celui de l'aménagement du territoire et de la politique de cohésion, l'idée qu'il existe non seulement des relations mais des interactions fortes entre le territoire communautaire et les territoires des pays voisins n'est pas encore intégrée. Les principaux documents de stratégie européenne en matière de cohésion et de développement régional continuent de prendre en compte uniquement l'espace communautaire. La réforme des fonds structurels et des instruments qui permettent d'organiser et de financer les relations avec les pays voisins apporte de sensibles améliorations en matière de coopération territoriale. Néanmoins, ces nouvelles réglementations ne permettent pas de dépasser certains problèmes et blocages pourtant identifiés depuis longtemps par les acteurs de la coopération territoriale avec les pays voisins. De plus, leur mise en œuvre intervient dans un contexte de baisse chronique de l'engagement financier de l'Union européenne dans son voisinage en matière d'aide au développement. L'efficacité des politiques européennes en matière de cohésion territoriale gagnerait à une plus grande coordination intersectorielle au sein de la Commission européenne.

Traditionally, the European Union sees itself as a closed entity in numerous fields. As far as the spatial planning and the cohesion policy are concerned, the existence of relations and even interactions between the European territory and the territories of the neighbouring countries are not taken into consideration. The main strategic documents in the fields of cohesion and regional development are almost exclusively focused on the territory of the European itself, without real consideration to the processes of globalisation and regionalisation. The recent reform of the structural funds and of the instruments dedicated to the relations with the countries of the neighbourhood is likely to bring sensible improvements especially in the field of the territorial cooperation. Nevertheless, these new instruments and regulations will certainly not be enough to solve some of the problems already underlined many years ago by the countries involved in the territorial cooperation with the neighbouring countries. Moreover, the expected improvements of this reform will be hampered by budget shortages : the disbursements of the EU 
(especially those of the member states) oriented to the countries of its actual geographical neighbourhood have been continuously decreasing since the 1960s. The efficiency of the European Union in the field of territorial cooperation would be dramatically enhanced by a stronger coordination of various DG of the European Commission (especially DG Regio and DG Relex).

INDEX

Mots-clés : Union européenne, développement régional, cohésion territoriale, frontières Index géographique : Europe

\section{AUTEURS}

NICOLAS GAUBERT

UMS Riate (CNRS-Diact - université Paris Diderot)

\section{YANN RICHARD}

UMR Ladyss (université Paris 1 Sorbonne) 